Meta

Journal des traducteurs

Translators' Journal

\title{
L'écriture littéraire bilingue : traduction ou réécriture ? Le cas de Salah Guermadi
}

\section{Salah Mejri}

Volume 45, numéro 3, septembre 2000

La traduction dans le monde arabe

URI : https://id.erudit.org/iderudit/003613ar

DOI : https://doi.org/10.7202/003613ar

Aller au sommaire du numéro

Éditeur(s)

Les Presses de l'Université de Montréal

ISSN

0026-0452 (imprimé)

1492-1421 (numérique)

Découvrir la revue

Citer cet article

Mejri, S. (2000). L'écriture littéraire bilingue : traduction ou réécriture ? Le cas de Salah Guermadi. Meta, 45(3), 450-457. https://doi.org/10.7202/003613ar
Résumé de l'article

L'expérience originale de l'écrivain tunisien Salah Guermadi, qui a publié certains de sesécrits simultanément en arabe et en français, représente une pratique permettant d'abandonner l'explication de la non-conformité des textes traduits et des textes originaux par l'attitude ou les compétences du traducteur. Puisqu'il s'agit d'un travail de réécriture, les textes de cet auteur fournissent l'occasion de chercher à identifier les éléments nécessairement différents d'un texte à un autre. C'est à travers l'étude d'une nouvelle que nous avons pu vérifier que plusieurs spécificités linguistiques échappent à toute opération traduisante (niveaux de langue, stéréotypie, croyances partagées, etc.). 


\title{
L'écriture littéraire bilingue: traduction ou réécriture? Le cas de Salah Guermadi
}

\author{
SALAH MEJRI \\ Université de Tunis 1, Tunis, Tunisie
}

\begin{abstract}
RÉSUMÉ
L'expérience originale de l'écrivain tunisien Salah Guermadi, qui a publié certains de ses écrits simultanément en arabe et en français, représente une pratique permettant d'abandonner l'explication de la non-conformité des textes traduits et des textes originaux par l'attitude ou les compétences du traducteur. Puisqu'il s'agit d'un travail de réécriture, les textes de cet auteur fournissent l'occasion de chercher à identifier les éléments nécessairement différents d'un texte à un autre. C'est à travers l'étude d'une nouvelle que nous avons pu vérifier que plusieurs spécificités linguistiques échappent à toute opération traduisante (niveaux de langue, stéréotypie, croyances partagées, etc.).
\end{abstract}

\begin{abstract}
The original experience of the Tunisian writer Salah Guermadi, who published some of his works both in Arabic and in French, provides an occasion to explaining the nonconformity of the translated texts with the original ones by the translator's attitude or skill. Since it is a work of rewriting, this writer's texts offer the opportunity to identify the elements which are necessarily different from one text to another. It is through the study of one of his novels that we have been able to realize that some linguistic specificities defy any translating process (language levels, stereotypy, shared beliefs, etc.).
\end{abstract}

\section{MOTS-CLÉS/KEYWORDS}

réécriture, traduction, arabe, français, stéréotypie

Il ne s'agit pas ici de la problématique inhérente aux traductions où il est vraiment très difficile de faire la part de l'auteur du texte initial et celle du traducteur dans le texte obtenu en $\mathrm{L}_{2}$. Nous voudrions dans ce qui suit interroger un type particulier de textes publiés par le même auteur en arabe et en français, ceux de Salah Guermadi, auteur et linguiste tunisien (1933-1982) connu par sa grande sensibilité au bilinguisme. Notre objectif, aussi paradoxal que cela puisse paraître, est d'apporter des éléments de réponse aux questions qu'on se pose devant les multiples transformations apportées aux textes traduits par les traducteurs professionnels.

Puisque, dans ce cas de figure, les textes sont rédigés dans les deux langues par le même auteur, il nous est théoriquement possible de dégager des différences qui ne relèvent ni d'une interprétation subjective du traducteur ni d'une liberté quelconque du traducteur vis-à-vis du texte initial. Si l'auteur réécrit son texte dans une deuxième langue dans un but fonctionnel, c'est parce qu'il est convaincu que les deux textes ne peuvent pas avoir la même teneur. Ce qui signifie que le même contenu (informationnel ou esthétique) du poème ou de la nouvelle change inéluctablement de configuration selon qu'on le verse dans un moule linguistique ou dans un autre. Autrement, cette opération de réécriture, si on ne tient pas compte de son 
intérêt fonctionnel, serait inutile. Réécrire ou traduire ses propres textes, c'est reconnaître en quelque sorte que l'idiomaticité, prise dans son sens premier, marque tellement les contenus au point d'en modifier la configuration finale. Cela revient aussi à dire que contenu et forme demeurent isolables.

\section{Que serait le contenu dans une nouvelle?}

Pour répondre à cette question, nous prenons comme exemple la nouvelle «Nocturnes ou pour l'amour d'Allah!» (1986: 37-56 en français ; 63-80 en arabe). Nous en dégageons la trame événementielle, les personnages, le cadre spatio-temporel, le mouvement narratif et les éléments diégétiques, éléments qui ne changent pas d'une langue à l'autre.

Il s'agit d'un pauvre mendiant qui a quitté depuis longtemps sa famille pour la capitale dans le but de chercher du travail. Il passe tout son temps à errer dans les rues de la ville et à mendier. L'auteur présente les événements survenus pendant le temps que dure l'histoire sous forme de tableaux: celui du mendiant discutant avec le serveur du bar qui finit par le laisser ingurgiter les restes des verres et des amusegueule; celui de la négresse qui chasse le mendiant parce qu'il fouille dans les poubelles qu'elle sort de l'immeuble pour chercher de quoi manger; celui du policier nettoyeur qui, après avoir pris son verre d'alcool, va au travail, mais tombe sur le mendiant et la négresse en train de se disputer. Il finit par l'arrêter et le conduire au poste. Suivent les scènes d'interrogatoire et d'hystérie générale.

Le tout est parsemé de notes humoristiques.

Si on considère que les invariants dans les deux textes sont tous ces éléments présentés dans le même ordre narratif, les transformations concerneront plutôt la forme. Nous entendons ici par forme non la manière dont le récit est fait mais la langue dans laquelle la nouvelle est rédigée.

\section{Les formes linguistiques}

À la lecture des deux textes, on est frappé par un contraste très net entre les deux nouvelles malgré l'identité du récit, des personnages et de la trame narrative. Chacune reflète un univers propre.

La question que nous voudrions poser porte sur l'origine de cette différence: est-ce le fruit d'un choix prémédité fait par l'auteur en vue de réaliser un type particulier d'exercice de style? Tel n'est pas le cas parce qu'il ne cherche à aucun moment à changer ni l'ordre de présentation des événements, ni celui des tableaux, ni celui des répliques. L'auteur suit scrupuleusement le même ordre dans les deux textes.

Est-ce plutôt dans la nature des commentaires et des incursions que l'auteur introduit dans le récit? Tel n'est pas non plus le cas parce qu'on note que l'auteur prend soin de faire apparaître dans les deux textes les réflexions humoristiques et les incursions de toutes sortes aux mêmes endroits et de la même manière.

Ce serait peut-être du côté du ton adopté dans la narration et des choix tropiques? Même si on ne dispose pas d'une définition précise du ton, on peut partir de l'usage des tropes dans les deux textes pour affirmer que l'auteur a cherché à utiliser scrupuleusement les mêmes figures et à user des mêmes raccourcis que les deux langues rendent disponibles. 
Si on ajoute toutes ces considérations aux invariants dégagés au début, seul le linguistique semble constituer le foyer principal des transformations (ou des différences) qui marquent chacun des textes et lui donnent un cachet propre et original.

\subsection{Le texte français}

Le texte français est écrit dans une langue accessible qui se distingue par une syntaxe qui évite les tournures alambiquées et tend vers une sorte de parataxe où prédomine, par moments, les phrases simples ou même des fragments coulés dans des matrices qui en assurent la cohérence:

Le mendiant regarde tout autour de lui, contemple la rue. La ville pour lui, est mystère et mystérieux le café. Leur café! Où les clients attablés devant des verres pleins et debout et des bouteilles vides et couchées sont tous sans visage. (p. 37)

Cliquetis des machines tapant le résultat des interrogatoires [...]. Bruits de mastication, d'ingurgitation et de lèvres qu'on pourlèche [...]. Nom, prénom, lieu de naissance, profession s'il y a lieu, nombre d'enfants, domicile s'il y a lieu, raison d'avoir rejoint la capitale, nombre de verres de vin bus, de poubelles farfouillées et de déchets et de papiers jetés sur la voie publique? [...]

Cliquetis des machines [...]. (p. 51)

Les dialogues sont rédigés dans une langue qui n'est pas spécialement marquée par des tournures syntaxiques propres à l'oral ou par un lexique familier prédominant. Même si l'auteur fournit l'effort d'assaisonner ces passages par certaines marques d'oralité, l'essentiel demeure écrit dans une langue non marquée. En témoigne cette tirade mise dans la bouche de "l'homme de peine du dispensaire»:

- J'ai entendu dire que les jumeaux sont nés le nez bouché. Et s'ils sont morts, c'est que l'on n'a pas trouvé quelqu'un pour monter la bouteille d'oxygène au premier étage. Elle est très lourde, cette bouteille et le monte-charge était en panne. Quant à l'infirmier de garde cette nuit-là, il ne pouvait pas la monter à lui tout seul [...].

Partant de ces repères, nous pouvons dire que le texte français, loin de rendre compte comme le fait le texte arabe, de l'atmosphère générale dans laquelle se déroulent les événements, limite les renvois culturels aux noms des personnages (Messaoud, le mari de la négresse; Meherzia, l'épouse du mendiant), à la mention de Dieu en arabe (Allah), à la dénomination d'une boisson locale (la boukha) et à d'autres éléments épars comme la «khémia» (= amuse-gueule) qu'on servait dans les bars de Tunis et les «Kentichis», une variété de dattes du Sud tunisien. Toutes les fois que l'auteur tente d'introduire des indications qui sont de nature à impliquer des faits culturels fixés dans la langue, il se heurte à la barrière des spécificités linguistiques.

Pour illustrer de telles limites, nous retenons les séquences suivantes: «la manne céleste» (p. 37), «Un de perdu, dix de retrouvés» (p. 41), «à l'impossible nul n'est tenu» (p. 43), «il fait un froid de canard» (p. 44), «je suis fauché comme un clou» (p. 44), «ventre affamé n’a pas de honte» (p. 47), « et après moi le déluge!» (p. 55), etc. De telles séquences qui n'existent pas telles quelles en arabe reflètent, indépendamment de la volonté de l'auteur des univers de croyances partagés par les locuteurs français, et leur emploi véhicule des stéréotypies fixées dans de telles expressions.

Il s'ensuit que le texte français, si on en effaçait les indications extralinguistiques dont le référent est étranger, dégagerait une atmosphère générale qui n'aurait pas 
forcément de rapports directs avec celle reflétée par le texte arabe équivalent. L'auteur, conscient des contraintes imposées par la langue, ne cherche pas à user de tournures qui traduiraient un tant soit peu les stéréotypies locales; au contraire, il met dans la bouche du mendiant, évoquant la cause du décès de sa petite fille, des déformations linguistiques qui n'ont aucun équivalent en arabe:

— Il [ = le médecin] prétend qu'elle avait attrapé la «poliomelette» ou je ne sais quoi?

— La poliomyélite, pas la «poliomelette», ignorant! (p. 53)

Devant des séquences fortement marquées comme certains refrains de chansons populaires, l'auteur n'opte par pour la même solution. Dans un cas, il traduit le texte arabe:

Sous les «Sribba»

Son pantalon

Sous les «Sribba»...

De ma charrette

J'en ai marre de ma charrette... (p. 55)

Dans l'autre, il se contente d'une simple translittération du texte d'origine:

Datchilou

Y a madamma, datchilou

Datchilou

Soub-il-boukha ou serbilou

Ya madamma (p. 55)

Ce qui trahit un désir réel chez l'auteur de faire communiquer la charge culturelle de l'univers dans lequel évoluent ses personnages, quitte à bloquer parfois la compréhension du contenu de la séquence. C'est ce genre d'incursion de fragments de discours translittérés dans le texte français, qu'ils soient des dénominations de réalités propres n'ayant pas de correspondants en $\mathrm{L}_{2}$ ou des séquences à grande charge culturelle, qui jouent le rôle d'élément d'ancrage du texte français dans une réalité autre que celle dénotée par le texte du récit. Cette fonction identitaire du langage trouve son expression dans l'usage de certaines niveaux de langue (l'argot, par exemple) dans les textes littéraires, usage qui peut entraîner des ruptures, parfois totales dans le décryptage du texte, tout comme ce qui se passe avec la translittération de passages empruntés à d'autres langues.

À la suite de ces indications, pourrions-nous avancer l'idée que le texte français serait en fait une réécriture du texte arabe? Pour apporter des éléments de réponse, il faudrait dégager les spécificités linguistiques du texte arabe et montrer comment elles serviraient de repères par rapport au texte français.

\subsection{Le texte arabe}

Malgré l'effort fourni par l'auteur pour transmettre le même contenu dans les deux langues, il est clair que l'usage de la langue lui impose des contenus extra-diégétiques transcendant le récit et son contenu strictement informationnel. En d'autres termes, le simple usage d'un code implique un ensemble de contraintes, de connotations et de valeurs linguistiques, difficile ou impossible à exprimer dans un autre code. Pour illustrer ce propos, nous retenons les trois aspects du texte arabe étudié: la diglossie, la stéréotypie et les charges culturelles non explicitées. 


\subsubsection{La diglossie}

L'auteur fait alterner dans le texte arabe littéral et arabe dialectal tunisien: le premier couvre la presque totalité du récit alors que le second tout l'espace discursif. Malgré les quelques éléments du lexique dialectal insérés dans le texte écrit en littéral, tous les passages du récit offrent au lecteur un texte littéraire rédigé dans une langue soutenue avec une syntaxe rigoureuse et un respect scrupuleux des marques casuelles telles qu'elles s'expriment, en l'absence de voyellation, dans le duel et le pluriel par exemple. S'opposent à cette écriture conforme à la tradition littéraire des passages, très nombreux, rédigés en dialectal, avec ce que cette orthographe non normée impose comme difficultés de déchiffrement du message par le lecteur.

C'est ce grand décalage entre ces deux niveaux de langue bien hiérarchisés qui détermine la configuration générale du texte arabe: il rythme la lecture du texte par les contrastes véhiculés par chaque niveau, l'un considéré comme langue de culture et de savoir, l'autre comme simple outil linguistique symbolisant l'ignorance et l'absence de formation, même minimale. Ces contrastes sont poussés à l'extrême par le fait même que le dialectal soit écrit; le simple fait d'écrire le dialectal semble relever du contradictoire dans la conscience linguistique collective. On peut voir dans cette tentative une position claire de l'auteur à l'égard de la question linguistique telle qu'elle se présente en Tunisie et dans d'autres pays arabes (cf. ici même Chadia Trabelsi, à propos de l'écrivain soudanais Taïeb Salah).

Par ailleurs, il est évident que le dialectal, en tant que langue maternelle, servant de vecteur à l'affectivité, traduit beaucoup mieux les réactions des personnages et reflète d'une manière plus fidèle les charges émotionnelles que l'expression linguistique spontanée véhicule.

Le dialectal véhicule, pour sa part, des spécificités linguistiques qui l'opposent au littéral et dont l'usage dans un contexte bilingue (arabe-français) conduit à une situation un peu originale. L'attitude vis-à-vis de l'emprunt est à cet égard très significative: le dialectal, comme toute langue d'usage courant, intègre n'importe quelle unité linguistique étrangère, pour peu qu'elle soit indispensable à la communication. Dans le texte que nous étudions un très grand nombre de termes d'origine étrangère, française ou autres (pour l'emprunt en arabe cf. Taïeb Baccouche, 1993), émaillent le texte en arabe littéral: /ba:r/ 'bar'; /oksizi:n/ 'oxygène'; /ruba/ 'robe'; /lasãsu:r/ 'ascenseur'; /kru:m/ 'chrome'; /babje bri:stu:l/ 'papier bristol'; /sirvi:s/ 'service'; /bu:ntu:/ 'un point'; /iljej/ 'il y est' (= un but); etc.

Le passage de l'arabe au français a pour conséquence logique la disparition de tout ce jeu linguistique parce qu'il n'est possible que dans le cadre du texte arabe: l'emprunt en tant que tel ne peut avoir de trace dans le texte français; les contrastes et les multiples valeurs dont les deux niveaux de langue de l'arabe ne sont pas non plus chargés.

\subsubsection{La stéréotypie}

Parmi les outils linguistiques de la stéréotypie, les séquences figées demeurent le support idéal où s'incrustent les visions partagées de la collectivité. Nous avons déjà cité quelques séquences françaises dont le contenu n'est pas forcément partagé avec l'arabe.

Aux intensifs français suivants, «un froid de canard» et "fauché comme un clou », l'auteur substitue des équivalents en dialectal : / $s$ sqontri:ne:zil/ litt. « Esqontri: ${ }^{1}$ » 'tombe'; /wxajjik jinza $\div$ mtabbis/, litt. «Ton frère, se dévêtit courbé.» On voit bien le 
chemin parcouru entre les univers partagés dans chaque langue. Le texte arabe ne se limite toutefois pas à de telles expressions: il renferme également des expressions et des termes dialectaux dont le transfert en $\mathrm{L}_{2}$ demeure impossible. Nous mentionnons à titre indicatif ces exemples:

- /jbe:tfi : da:r xa:ltu :/, litt. «Il passe la nuit à la maison de sa tante.»; le sens global: «Il passe la nuit en prison.»

- /mharhra trakkib sitta wsitti:n ki:f/, litt. «piquante, procurant soixante-six 'kifs'»; le sens global: «très piquante».

La stéréotypie n'est pas limitée aux expressions idiomatiques: elle peut avoir comme support les stratégies d'échanges discursifs qui présupposent l'emploi d'éléments linguistiques marquant des rapports précis entre les interlocuteurs. Le tunisien dispose, entre autres outils, d'une forme diminutive de [sidi] (monsieur), [swidi] dont la charge dépréciative, curieusement, ne porte pas sur la personne dénommée par le vocatif, mais sur l'utilisateur de ce diminutif.

Les exemples de spécificités linguistiques dont le texte arabe est chargé sont très nombreux; nous pouvons en ajouter les modalisations véhiculées par les expressions de salutation, les dénominations appréciatives, etc.

\subsubsection{Les charges culturelles non explicitées}

Nous voulons signifier par là toutes les charges connotatives et toutes les valeurs liées aux différents usages du lexique et à toutes sortes de tournures et de formations, qu'elles soient d'origine syntaxique, phonétique, lexicale ou sémantique. Parmi les données de contenu n'ayant pas de contrepartie (= signifiant) tangible, c'est-à-dire n'ayant pas de consistance phonétique ou formelle, nous indiquons un certain nombre de faits dont l'origine n'est qu'un écho ou une allusion ou une suggestion diffuse:

a) L'usage de la langue n'est pas indifférent aux situations particulières. Ainsi en est-il du mot «chérie »: il s'agit d'un emprunt fait par le dialectal tunisien au français, n'ayant pas de connotations particulières à l'origine, qui se trouve investi, en plus du contenu dénotatif, d'une charge sémantique dont le contenu pourrait être traduit comme suit: "l'appartenance à une frange sociale dont le snobisme frôle le ridicule». Puisque l'auteur emploie le même terme en arabe, il ajoute au texte arabe cette connotation qui déborde le terme français emprunté.

b) /mu:t rabbi :/, litt. «la mort de Dieu» pour signifier «mort naturelle», est une autre expression qui impose, rien que par sa littéralité, des échos de nature surnaturelle, qu'on ne soupçonne pas dans la séquence française qui ramène la mort à un mécanisme plutôt admissible dans une logique «évolutionniste».

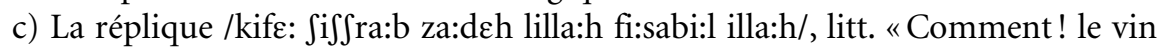
aussi, c'est pour l'amour d'Allah!?» (p. 38, texte français) ne renvoie nécessairement pas à une contradiction en français, puisque le vin, en tant que boisson, n'est pas considéré comme un fait interdit, alors qu'il a le statut de péché dans le contexte musulman : associer une quête dans la bouche d'un mendiant à une boisson alcoolisée est un sacrilège!

d) «Messaoud! N'oublie pas le cinquième, ils sont rentrés de vacances » est une tirade qui peut paraître d'un contenu sémantique neutre. Or, placée dans ce contexte précis où il est question d'une misère effroyable, où mendiant et concierge s'opposent comme deux mondes hiérarchiquement opposés, elle charge le mot «vacances» 
d'un contenu mélioratif qu'il ne véhicule pas dans un message courant, désignant des gens aisés pouvant profiter des vacances.

En plus de ces indications, il y a lieu d'attirer l'attention sur le grand nombre d'échos sémantiques que le simple usage d'un code, en l'occurrence le dialectal tunisien, active et met en branle pour déterminer la configuration finale du message

\section{Le strictement linguistique}

Lorsqu'un auteur tient à réécrire le même texte dans deux langues différentes, sans qu'il y ait un besoin apparent pour le faire, il traduit par là un sentiment de linguiste qui verrait dans le transfert d'une langue à une autre du même texte narratif une opération double où le contenu informationnel serait identique mais où des disparités intrinsèquement linguistiques déterminent la configuration finale du texte, parmi lesquelles nous retenons :

- le niveau strictement phonologique qui est susceptible de mettre en œuvre des mécanismes de sonorité et de concordances rythmiques spécifiques (que nous n’avons pas retenues ici pour notre analyse);

- le niveau lexical, qui est de loin le plus suggestif, et qui fournit des repères d'analyse suffisamment pertinents, pour mettre en relief l'originalité de chaque texte et la charge connotative à chaque type de code; l'emploi du code déterminant un ensemble de renvois connotatifs qui n'obéissent pas à la volonté de l'usager, dès qu'il choisit ce code pour s'exprimer;

- la mise en valeur des contenus pragmatiques qui s'inscrivent dans le cadre des usages fixés dans chaque code;

- les jeux chargés de significations propres et permis dans chaque code.

\section{Conclusion}

À la suite de cette démonstration, nous voudrions attirer l'attention sur le fait que la réécriture du même contenu diégétique (= événementiel) est loin d'être un simple transfert de code; il implique, par le simple changement de code, des transferts d'univers de croyances partagés, des stéréotypies fixées dans les dénominations de chaque code et toute la charge culturelle qui transcende le code linguistique dans son intégralité.

Ainsi la traduction se transmue-t-elle en réécriture, c'est-à-dire une reprise à la fois identique et autre, l'autre confirmant l'identique dans une dialectique de création à laquelle l'auteur traducteur ne peut échapper.

\section{NOTE}

1. Ne disposant pas de dictionnaire étymologique du dialectal tunisien, nous avons procédé à une enquête auprès d'informateurs pour déterminer le sens du mot employé isolément, mais il s'est avéré que personne n'en connaît ni l'origine ni le contenu propre. Il doit son existence à l'expression dans laquelle il figure. 


\section{RÉFÉRENCES}

\section{A. Écrits de Salah Guermadi}

\section{Nouvelles et théâtre}

Guermadi, S. (1961-1962): «Saïd ou le grain de Halfaouine» [en arabe], Ettajdi:d, Tunis.

- (1986): Le frigidaire, Tunis, Alif, un recueil de nouvelles comportant:

- en français: «Nocturnes ou pour l'amour d'Allah», "Le cireur », "Le frigidaire», "Bonne mine», «La salive-à-lèche», «Le territoire humain».

- en arabe: «Almata, mata» [c'est nous qui traduisons], «Le cireur», «Nocturnes ou pour l'amour d'Allah».

— (1975-1976): «Poisson Halaoui», pièce de théâtre, Tunis, Alif, no 6 (1975), p. 98-126, et no 7 (1976), p. 50-79.

\section{Poésie}

- (1970): Avec ou sans, Tunis, Cérès production ( $2^{\mathrm{e}}$ éd.: 1982).

_ (1975): Nos ancêtres les bédouins, Paris, coll. «Pierre Jean Oswald».

\section{B. Références générales}

Awaiss, H. et J. Hardane, dir. (1999): Traduction: approches et théories, Beyrouth, Université Saint-Joseph.

BAccouche, T. (1994): L'emprunt en arabe moderne, Tunis, Beit Al-Hikma - Carthage et IBLV.

Baccouche, T. et S. Mejri (1993): Salah Guermadi et le plurilinguisme (en arabe), Tunis, Publications de la Faculté des Lettres de la Manouba.

Baccouche, T., S. Mejri et A. Clas, dir. (1998): La mémoire des mots, vol. II, Revue Tunisienne des sciences sociales, $\mathrm{n}^{\circ} 117$, Tunis, CERES.

Baccouche, T. et S. MejRI, dir. (1998) : L’information grammaticale, numéro spécial «Tunisie».

Clas, A., S. Mejri et T. Baccouche (1998): La mémoire des mots, Actes du Colloque de Tunis, Actualité scientifique, Paris, AUPELF-UREF.

Goulet, A., dir. (1994): Le stéréotype, Caen, Presses universitaires de Caen.

Gross, G. (1996): Les expressions figées en français, Paris, Ophrys.

LAMbert, J. et A. Lefevere, éd. (1993): La traduction dans le développement des littératures: Actes $d u X I^{e}$ Congrès de l'Association internationale de littérature comparée, Berne, Peter Lang, Bern.

MejRI, S. (1997): Le figement lexical: descriptions linguistiques et structuration sémantique, Tunis, Publications de la Faculté de Lettres de la Manouba.

Mejri, S., G. Gross, A. Clas et T. Baccouche, dir. (1998): Le figement lexical: Actes de la $1^{\text {re }}$ $R L M$, Tunis, CERES.

Truffaut, L., dir. (1999) : La traduction professionnelle. Former et perfectionner, Lausanne, Institut Étienne Dolet. 